\title{
A DEFERRED ACCEPTANCE MECHANISM FOR DECENTRALIZED, FAST, AND FAIR CHILDCARE ASSIGNMENT
}

\author{
Tobias Reischmann
}

University of Münster, Germany

tobias.reischmann@uni-muenster.de

Thilo Klein

Pforzheim University and ZEW - Leibniz-Centre for

European Economic Research, Germany

thilo.klein@hs-pforzheim.de

Sven Giegerich

University of Oxford, United Kingdom

sven.giegerich@oii.ox.ac.uk

\begin{abstract}
We design a program-proposing deferred acceptance mechanism with ties (DAT) and apply it to childcare assignment in two German cities. The mechanism can accommodate complementarities in providers' preferences, is fast to terminate even in larger cities, is difficult to manipulate in practice, and produces stable allocations. It can be further sped up by introducing two new features. First, allowing for an arbitrary share of facilities who participate in a centralized manner by submitting a rank-order-list over applicants. Second, by breaking ties in applicants' rank-order-lists on a first-come-first-serve basis, which sets incentives for programs to propose faster. We provide and evaluate simulation results.

All authors have contributed equally. We acknowledge helpful comments from Péter Biró, Inácio Bó, Tobias Riehm and audiences at the 14th and 15th Matching-in-Practice Workshops in Cologne and Mannheim. We are thankful to the youth welfare office of the district of Steinfurt for their cooperation in the project. We acknowledge funding from the Leibniz Association as part of project K125/2018: Improving school admissions for diversity and better learning outcomes.
\end{abstract}

Copyright (C Tobias Reischmann, Thilo Klein, Sven Giegerich / 6(1), 2021, 59-100. 
Keywords: Childcare assignment, deferred acceptance algorithm, simulation. JEL Classification Numbers: C78, D02, D47, D82, I24.

\section{INTRODUCTION}

$T^{N}$ many German cities, the allocation of available childcare placements Iis not transparent and carries considerable costs for parents and childcare providers alike. While there is a recognized shortage of childcare placements, inefficient allocation procedures have made the shortage seem more acute than it actually is. ${ }^{1}$ One possible blanket solution to the problem would be to introduce a central allocation system that relies on tried and tested matching algorithms, thus ensuring a well-designed matching system.

Online platforms already facilitate the registration for childcare placements in Germany. ${ }^{2}$ Their scope, however, is often limited to the registration of applications and their forwarding to childcare providers. Very few platforms allow for a coordinated offer process that takes into account both parents' and providers' preferences. Instead, childcare providers send out offers independently, in an uncoordinated fashion. Problems thus arise when (i) parents feel forced to accept an early unattractive offer for the sake of security or (ii) when they temporarily hold and thus block placements for other families in anticipation of a better offer. The first aspect can lead to what we will refer to as an unfair admission. The second aspect slows down the admission process, resulting in uncertainty on the side of parents and employers.

One solution to these problems would be to establish a "central clearinghouse" for admissions. Such clearinghouses have proven their worth in grade school and university admissions in Germany and other countries worldwide. However, they take time to develop. For example, five years after its creation, the German clearinghouse for university admissions only had a market share of $13 \% .{ }^{3}$ Furthermore, similar institutions have yet to emerge for childcare markets, a fact that we attribute to small care-group sizes, the large share of private (rather than public) providers, and complementarities in provider preferences.

1 Several illustrative newspaper articles raise these issues (e.g. Bös, 2017; Völker, 2018).

2 An example is the 'Kita-Navigator' software, which is widely used in the state of North Rhine-Westphalia.

3 According to Konegen-Grenier (2018) in 2017 only 1,080 out of 8,097 academic programs with restricted admissions used the central clearing house for universities in Germany (Gehlke et al., 2017). 
Private providers are often not able or willing to contribute full ranking lists of children to a central clearinghouse. To account for these market details, we depart from the literature by proposing an admission mechanism that allows private providers to make decentralized offer decisions while providing fast and fair results. The matching literature has only recently reflected an interest in such decentralized mechanisms; see for example, Bó \& Hakimov (2016), who focus on strategy-proof design for college admissions, and Grenet et al. (2019), who focus on applicant-side complementarities (i.e. friends preferring to study at the same university). By contrast, the IDAT mechanism proposed in this paper focuses on a setting in which complementarities are on the provider side. The IDAT accommodates these complementarities and speeds up the deferred acceptance mechanism in this decentralized setting. We believe that this model is a good intermediate step in moving from a decentralized market to a fully centralized mechanism. In the municipalities that adopted the mechanism, market coverage was $100 \%$ from year one.

As a starting point, we take the current allocation practice in the city of Münster in North Rhine-Westphalia (NRW), the most populous German state. This allocation practice is the most commonly used method in large cities in NRW and other German states (Klein \& Herzog, 2018). Admissions are for a fixed start date at the beginning of the school year in August, when most childcare placements free up. While we take into consideration dynamic aspects that arise when parents move or change placements (Kennes et al., 2014), these factors are of secondary importance in this context.

The matching literature has established speed and fairness as conflicting desiderata when allocation mechanisms are used in a decentralized context. Fairness can be defined in terms of the stability of an allocation. An allocation is stable if and only if it is non-wasteful and no market participant has justified envy of another participant (Kamada \& Kojima, 2020). Justified envy occurs if an applicant would prefer a place at facility $A$ but receives a childcare place at facility $B$, while later learning that an applicant with a lower priority received an offer from $A .{ }^{4}$ One of mechanisms that satisfy this requirement is the deferred acceptance (DA) algorithm (Roth \& Sotomayor, 1992). This algorithm allows applicants to defer the acceptance of an offer until all higher-ranked childcare facilities have been considered. While widely

${ }^{4}$ In the childcare context, priorities are established based on admissions criteria, such as geographical distance, socio-economic status, single parenthood, siblings attending the same facility and the parents' occupational status. 
used in centralized markets (Biró, 2017, for a recent overview), the DA is slow to complete matching in decentralized markets. To speed up the process, many childcare providers make exploding offers that only remain valid for two weeks. However, exploding offers force applicants to accept early, unattractive offers (for the sake of security) and are thus considered unfair.

In this paper, we introduce a deferred acceptance mechanism with ties (DAT). The mechanism runs in multiple iterations to include the decentralized offer decisions of private facilities. Compared to running a standard DA in a decentralized context, we accelerate the process by:

(i) automating parents' acceptance decisions based on their submitted rankorder preference of childcare facilities,

(ii) automating public facilities' offer decisions based on pre-specified rules (e.g. admissions criteria), and

(iii) incentivizing private facilities not to delay making offers.

The third element is implemented by allowing parents to state weak preferences (i.e. in-differences) and prioritizing competing offers from the same indifference class on a first-come, first-served basis.

The new mechanism allows one to cater to the interests of all involved stakeholders, including parents, childcare providers, and cities. For parents, fast assignment is desirable for planning reentry into the labor market. Furthermore, fairness is desirable for parents who value transparency. For childcare providers, a fast procedure reduces planning uncertainties, and fairness ensures that providers' admissions criteria are respected. Finally, for the city, a fast process has positive labor market effects, and fairness improves the perception of an efficient public administration, while also reducing the risk of lawsuits.

The mechanism, which is implemented as a software application, was tested in the cities of Saerbeck and Greven (NRW). We also evaluate the applicability and scalability of the mechanism in larger markets.

The remainder of the paper is organized as follows. Section 2 illustrates the context of childcare assignment in the city of Münster and presents the four challenges identified for the design of a revised assignment process. In section 3, we review the literature while focusing on our identified design 
restrictions. Section 4 presents the proposed mechanism, its properties and software implementation. In section 5, we evaluate the mechanism with respect to its implementation in the two cities and we present simulation evidence on its scalability for larger cities. Section 6 concludes.

\section{THE GERMAN CHILDCARE MATCHING PROBLEM}

\subsection{Context}

In 2013 legal changes in Germany granted children under the age of three a legal right to a childcare placement. This change in legislation has generated extremely high demand and strong competition for available placements. In Germany, cities are responsible for designing their childcare market and implementing admission processes in consultation with providers. One of the characteristics of the German childcare market is provider autonomy, which gives the provider the right to choose their own admission criteria and decide which children are granted a placement at their facility. The admission process thus differs between cities and regions (Klein \& Herzog, 2018). In the following, we outline the process in the city of Münster and highlight the problems associated with designing an admission process that fulfills the requirements of all stakeholders.

The city of Münster has a population of 314,000 and 190 childcare facilities. About 15\% of the facilities are public (i.e. held and managed by the city). The other facilities are run by different social service providers, such as the church, the German Red Cross, and parental initiatives. Münster conducts one single admissions process every year. The process begins in November for placements starting in August. In November, most facilities organize an open day for parents to get to know their facility and staff. Parents can then register a place for their child online up to the registration deadline on February 1 st. This is done by listing up to seven acceptable facilities, without ranking them. ${ }^{5}$ The city has published universal admission criteria to guide providers. However, providers neither need to comply nor publish their own admission criteria on the internet platform, which creates uncertainty for parents when registering their choices. After the registration deadline, facilities decide which applicants to send an offer to. This is an uncoordinated process, although some facilities

5 In the last years, this was extended to 12 acceptable facilities including in-home daycare providers. 
may coordinate their offers on an informal basis. Offers are exploding, and a decision is generally expected within two weeks. If parents accept the offer, they are removed from the system and marked as no longer available for other facilities. If the offer expires or is declined, facilities send an offer to the next applicant in line. This process takes about four months.

\subsection{Method}

The following analysis of the childcare market in Münster gathers the insights drawn from a case study that comprises multiple information sources. These sources include direct information about the process from the city, semistructured interviews with a representative selection of childcare providers, a survey among parents, as well as several newspaper articles.

Five interviews were conducted with individuals responsible for the application process at the facilities. A subset of individuals among all providers was chosen due to the heterogeneity between providers in terms of the number of childcare facilities under management, the provider's organizational structure, and hierarchical level at which application decisions are made. The interview group contained the head of a municipal childcare facility, the head of a Protestant childcare facility, the head of a Catholic community, a divisional director of the German Red Cross, and the head of a parental initiative. The interview questions focused on the current admission process as well as requirements for the redesign of the mechanism.

The survey was conducted among parents who had participated in the childcare assignment in past years or who planned to do so during the next assignment. The survey contained questions about their preferences. They were asked about their registration decisions during the assignment, as well as their actual preferences. Furthermore, we asked about the importance of factors influencing these preferences, such as a facility's geographical distance and quality. Finally, the parents were asked to report on different characteristics known from the interviews to influence the facilities' priority rankings, such as the age of the child or the parents' denomination. Unfortunately, despite a large marketing campaign with flyers and posters in every facility, only 295 complete survey responses were received. Considering that over 3,500 parents apply for a childcare placement every year, this sample is not large enough to be used in statistical analysis or simulations. However, it can still provide insight into the preferences held by parents. When specific questions from the 
survey are mentioned in the following, they are referenced in the footnotes.

\subsection{Problem Description}

From the gathered information sources four major problems and challenges could be identified in total. The first one is perceived or effective unfairness. This problem arises when there is a lack of transparency concerning how admissions criteria are applied or how parent preferences are taken into account. The second challenge is associated with the lengthy time requirement of the current process, which causes insecurities on both sides of the market. A third challenge is posed by provider autonomy, which makes it impossible to centralize the entire application process, and which establishes important requirements for decentralized decision-making. Finally, the heterogeneity of the childcare placements represents the fourth challenge. More specifically, requirements regarding group composition and variety complicate the application of standard theory, and make adjustments necessary.

\subsubsection{Fairness}

Req 1.1 - Perceived Unfairness: One reason for perceived unfairness in the admission process stems from intransparency concerning how admissions criteria are applied. Although the admissions criteria of most facilities are accessible online, if and how the facilities implement these criteria is not certain or transparent. Thus, there is a risk that decisions will be unduly influenced by subjective factors, which is perceived as unfair by the parents. Indeed, in a 2017 court case, the city of Münster was criticized by the court for a lack of transparency (OVG NRW, 2017).

Req 1.2 - Importance of Rankings: Parents are not able to accurately state their preferences. In current practice, parents are only able to specify an unranked set of childcare providers or in-home daycare. It is only possible to differentiate between the options by specifying a priority for childcare or in-home daycare. Thus, parents cannot indicate from which childcare facility they might prefer to receive an offer. This problem was raised throughout the interviews and in local newspapers.

Req 1.3 - Exploding Offers: The only other way for parents to express their preferences in the admissions process is by deciding if they want to accept 
a proposed offer made by a childcare facility. However, in this way, parents are not always able to voice their true preferences concerning facilities, since often they are confronted with incomplete information. In particular, when receiving an exploding offer with a two-week response deadline, they have no idea if a better offer might arrive at a later point in time. One of the interviewed individuals reported a case in which parents would have received a better offer after accepting a worse one. Their early acceptance of the inferior offer was due to their urgent need of a placement and uncertainty as to whether they would receive another offer if they declined the first.

Req 1.4 - Ability to Specify Rankings: In order to apply the parents' preferences efficiently during the admissions process, we would need to gather their true preferences a priori. Our survey revealed that parents often state several preferred facilities in their application and have no problem ranking them. Furthermore, parents often diverge in terms of the cardinal utility they derive from different childcare facilities. ${ }^{6}$

\subsubsection{Speed}

Req 2.1 - Time Requirement: The lengthy time requirement of the current process is yet another challenge. This time requirement is inconvenient for all stakeholders and also causes a substantial administrative burden for the facilities. The length of the process is mainly caused by the lack of coordination between the providers, combined with a reliance on exploding offers. One interview participant reported that they actively advised parents to make use of the full duration of the exploding offer since they might receive a better option within that time frame. Such behavior, of course, delays the entire application process, which often stretches over several months in practice.

6 In our survey, the parents were asked within two questions about their preference structure. First, they were asked to order their facilities in terms of preference. Second, they were asked to assign a point value from 0 to 100 to each facility, representing how they would value a placement at each facility. The first priority was fixed at 100 points. On average, the parents ranked 3.4 facilities while assigning the lowest-ranked facility a point value of 66 . This average also included many parents that only listed one facility. Others differentiated strongly in terms of cardinal utility, using the full range down to a value of 0 . It also could be observed that parents often chose divergently sized "steps" in cardinal utility when ranking multiple facilities. 
Req 2.2 - Uncertainty: Ultimately, the extensive duration of the process leads to uncertainty for parents and childcare providers alike. Parents need to know if they have received a placement for their child and thus if they can go back to work. The providers suffer financial insecurity since the number of vacant placements directly influences their financial resources. Consequently, they need to know the number of accepted children, in order to calculate their staff requirements accurately. This problem was mentioned by the two smaller childcare providers, which are most affected by financial insecurity.

Req 2.3 - Administrative Burden: In general, providers complained about a massive administrative burden during the application phase. A new mechanism could reduce this workload by automating decisions based on a priori ranking lists. Such automation could both reduce the duration of the process and administrative costs.

Req 2.4 - Problems with Existing Mechanisms: However, existing mechanisms from theory, such as the DA, require the preference rankings of both the parents and the providers to be known a priori by the central clearinghouse. In the interviews, concerns were raised that it is not possible or practical for some facilities to place all their applications in one ranking list. One such argument was made by the parental initiative. Due to high parental participation at the facility, they seek to interview each family in advance of the assignment process. Therefore, it is impractical for these providers to rank the full set of applicants. Furthermore, each application interview takes time, thus influencing how the facilities' preferences can be obtained and used.

\subsubsection{Provider Autonomy}

Req 3.1 - Provider Autonomy: Provider autonomy, that is, the right of each provider to define and use their own application criteria, poses the third challenge. According to this principle, providers may independently decide on which children they wish to accept. This autonomy, which is granted to all privately run providers, is enshrined in law, and thus represents an essential requirement. Thus, we cannot force the facilities to provide global preference lists.

Req 3.2 - Adoption Probability: Furthermore, it will not be possible to implement a new system without the approval of the providers. The opinions among the interviewed persons in this regard were quite diverse. The prospect 
of an automated system was particularly welcomed by providers that strive to implement entirely objective admissions criteria. Others expressed various reservations, ranging from a perceived loss of power over the decision process to difficulties in handling certain group compositions. They also cited difficulties connected to edge cases, such as the requirement to match children with special needs to the right facilities.

\subsubsection{Heterogeneity of Placements}

Req 4.1 - Scope of Daycare: Finally, the heterogeneity of offered placements represents a fourth challenge. Specifically, providers offer different forms of childcare. In Münster, these are 25h, 35h and 45h per week. As a consequence, providers have several types of childcare placements. The preferences of parents might differ for the same facility depending on the type of placement. Also, the ranking lists of the providers might differ for different placements for instance, due to the group composition, if the placements belong to different playgroups within the facility.

Req 4.2 - Group Composition: Group composition is another argument as to why ranking lists are not known a priori. All five interviewed providers mentioned this issue, although it varied in importance from major to minor. Providers seek to guarantee that every child has a playmate; in this regard, age and gender play an important role. While childcare providers run by the municipality have a legally binding requirement to accept older children first, the others (such as the parental initiative and the Protestant church) stated that they considered both the age and gender of the child during the acceptance process for a specific placement. Some providers even aim to reach a 1:1 gender ratio within a playgroup. Thus, if group composition influences the formation of ranking lists, those lists might change during the allocation process, depending on current status of placement allocations at the same facility. This conflicts with the application of a standard DA, which requires unchanging ranking lists stated a priori.

\section{RELATED LITERATURE}

In this section, we briefly review the literature, focusing on studies that discuss mechanisms that fulfill one or more of the requirements identified in our problem analysis. 
The childcare market is a two-sided market (Roth \& Sotomayor, 1992), where both parents and facilities care about the outcome of the matching process. Requirement 2.3.4 showed that facilities care not only about respecting their admissions criteria (which seek to ensure stable placement allocations), but they also have strong preferences concerning group composition.

There is a growing literature on two-sided matching markets in childcare assignment (Veski et al., 2017; Carlsson \& Thomsen, 2014; Kennes et al., 2014). The workhorse for most applications is the well-studied DA. While the applicant-proposing DA is strategy-proof for applicants (but not for programs), the program-proposing DA that we build on in this paper is neither strategy-proof for applicants nor for programs (Roth \& Sotomayor, 1992). In practice, however, the latter is also difficult to manipulate and widely used, e.g. in German university admissions (Braun et al., 2010). Azevedo \& Budish (2018) substantiate this observation theoretically by arguing that manipulation incentives disappear in the DA as the market size grows, such that the DA is "strategy-proof in the large" (SP-L).

There are various ways to allow facilities to maintain control over group composition when using the DA mechanism (see Requirement 4.2). The simple solution is to use strict admissions quotas. These are implemented in the form of so-called slot-specific priorities (Kominers \& Sönmez, 2016). To achieve, say, a gender-balanced intake, the childcare facility splits its slots (i.e. placements) into two halves. In the first half, girls have priority over boys. In the second half, priority is given to boys. This guarantees a balanced gender intake if there is sufficient demand from both sexes. ${ }^{7}$

In general, the DA can incorporate more complex distributional requirements (Gonczarowski et al., 2019). Refined lower and upper quotas for slots for example, to balance assignments according to socioeconomic characteristics - have been discussed in the literature with a view to affirmative action policies (Nguyen \& Vohra, 2019; Hafalir et al., 2013; Kojima, 2012). Furthermore, Sönmez \& Yenmez (2020) show that so-called choice functions for facility preferences can guarantee maximum compliance in the event that applicants qualify for multiple reserved slots (e.g. a disabled girl in combination with slots reserved for gender and disability).

Another topic is the inclusion of capacity constraints in the mechanism. Kamada \& Kojima (2020) discuss the issue of staff headcount requirements

7 Aygün \& Turhan (2020) show how to design a strategy-proof transfer scheme, if capacities are not fulfilled in certain slots 
based on the age of the children. This criterion can be modeled in a mechanism such that one child occupies several slots depending on its age. In Germany, regulations define the required staff to child ratio. ${ }^{8}$ However, the number of placements each childcare facility can provide per age category is determined in cooperation with the municipality prior to the allocation, since numerous factors influence this decision. ${ }^{9}$ If the legal restrictions applied to slot assignment are eased at some point in Germany, it would be interesting to integrate into our research flexible slot management based on the work of Kamada \& Kojima. Until then, however, we must consider as discrete the markets for children above and below the age of three.

The requirements described in our problem analysis, however, go beyond the implementation of quota rules. Firstly, Requirement 3.1 states that private facilities are reluctant to disclose their preferred group composition to a central clearinghouse. Therefore, the implementation of choice rules only seems feasible for public providers. Second, preference complementarities arise from staff-to-child ratio requirements based on a child's age. A simple example of a complementary preference is when matching is for a single applicant or for a couple (Roth \& Peranson, 1999). In the case of childcare, singles are equivalent to children above age three, and couples are equivalent to children below three (as the latter require more intensive care, and can thus be viewed as occupying two slots). Even in this basic setting, the existence of stable matches is no longer guaranteed. ${ }^{10}$ The analysis of matching based on more general complementarities also arrived at several negative results (Kamada \& Kojima, 2018; Delacrétaz et al., 2016).

Integer programming is an alternative approach to solve matching problems with distributional constraints (e.g. Ágoston et al., 2018; Geitle et al., 2020). However, in our context the constraints of private childcare providers are not known to the matchmaker, making integer programming not viable (see Requirement 3.1).

8 Children aged one to three require a ratio of 1 childcare worker per 10 children, while children above the age of three require only a ration of 1 to 20 .

9 Younger children require a place to sleep over noon within the facility. Thus, the available slots depend on the planning of the facilities room capacities, which has to be documented and approved by the municipality, first.

${ }^{10}$ The Roth Peranson algorithm (Roth \& Peranson, 1999) and an SAT-solver (Drummond et al., 2015) can find a stable matching in this setting if it exists. However, the existence of a stable matching is unlikely for markets with a large number of couples (Kojima et al., 2013), and the existing algorithms do not fit the requirement of a decentralized matching procedure. 
In light of the limited ability of centralized admissions schemes to accommodate general complementarities, we thus consider decentralized mechanisms. The matching literature has only recently reflected an interest in such mechanisms. Bó \& Hakimov (2016) demonstrate how an iterative version of the applicant-proposing DA - in which students make applications one at a time - helps students to learn about their feasible set of schools. Similarly, the DoSV mechanism used for German university admission also combines dynamic steps with a final DA phase, but is based on the program-proposing DA. The DoSV differs from the DA in that the centralized DA is preceded by a decentralized phase of 34 days, where programs make admission offers to their preferred students in real time. Students, in turn, can decide to accept an offer and exit the process, to retain all offers, or to keep just a subset of offers. In the subsequent centralized phase, students who have not yet exited the process can reconsider their rank-order lists and participate in the programproposing DA (see Grenet et al., 2019, for a detailed description and analysis).

The DoSV mechanism has several advantages. It relaxes the assumption of fixed student preferences by allowing students to form preferences during the admissions process and can accommodate (to some degree) complementarities in the form of friends preferring to study at the same university. Instead, the focus of the IDAT mechanism - as proposed in this paper - is a setting where complementarities are on the program-side and a centralized phase is ruled out by institutional constraints. IDAT accommodates these complementarities and speeds up the DA in this setting.

A series of papers also make use of a family of mechanisms characterized by Chen \& Kesten (2017) that result in allocations between the DA and the immediate acceptance (IA) algorithm. One example is the adaptive IA (Mennle $\&$ Seuken, 2017), which allows schools in each round to condition their admissions based on admissions already confirmed in the previous rounds, while at the same time making it safer for students to report their true preferences. Several other papers also study dynamic matching procedures inspired by college admissions practices (Echenique et al., 2016; Gong \& Liang, 2017; Klijn et al., 2019). The IDAT mechanism proposed in the next sections has, to the best of our knowledge, not yet been studied or applied in practice.

An additional feature of the adaptive DA mechanism we propose below is that it allows for weak preferences, i.e. indifference classes, which are used to incentivize providers not to delay their offers (see Requirements 2.3.2). Indifferences result in an efficiency-loss because providers could be made 
better-off by swapping their assigned applicants. This source of inefficiency can be improved on by allowing for such swaps using so-called "stable improvement cycles" (Erdil \& Ergin, 2008). ${ }^{11}$ We will not discuss this issue below because a shortcoming of such post-hoc improvement cycles is that they induce additional strategic behaviour.

Furthermore, there is literature about matching with contracts, which shows that market agents can be matched using different kinds of assignments (Hatfield \& Milgrom, 2005). At first glance, the outlined model of the childcare assignment problem qualifies as a form of matching with contracts, since the different scopes of care that are offered by the providers could be mapped as different contracts with the same facility. However, in German cities, the number of placements a facility can offer for each scope of care has to be fixed a priori in consultation with the city due to various factors (e.g. security regulations or room planning). For this reason, we chose the more comprehensible alternative for representing divergent scopes of care programs by separating the placements in each facility, which is possible since each care program has its own capacity limit and might also have a distinct preference ranking. Also, the advantages inherent in the theory about matching with contracts, such as including monetary transfers, are not relevant to our specific use case. If the IDAT were to be applied in the future to a market in which placements regarding the scope of care are not fixed but interchangeable, our model could be extended to include matching with contracts.

\section{SOLUTION}

\subsection{Mechanism}

The market for childcare placements represents a two-sided matching market. In our model, children are on one side of the market. All children $I=\left\{i_{1}, \ldots, i_{N}\right\}$ are single individuals. On the other side of the market, we have the providers $S=\left\{s_{1}, \ldots, s_{N}\right\}$. Each facility is run by a provider. These providers can be categorized into those who want to provide a complete list of priorities (for automation) and those that prefer to make offers conditional on the status of previous offers. For simplicity, we refer to the facilities run by the first kind of provider as public providers $S_{P u b}$ and the facilities run

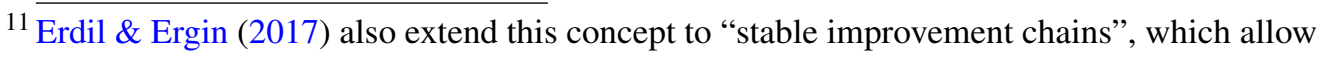
for indifferences on both sides of the market. 
by the second type as private providers $S_{P r}$ so that $S=S_{P u b} \cup S_{P r}$ as well as $S_{P u b} \cap S_{P r}=\emptyset$.

For educational purposes, providers usually separate their children into multiple play groups. Often, each group has different characteristics, such as the scope of care (in hours per week) or an age limit. These characteristics influence the preferences of the parents and can also result in a differing priority list among one facility type. Additionally, each play group has a capacity constraint in terms of a limited number of available placements. For these reasons, these groups are used as entities on the facility side within the mechanism. Further, these entities are called "programs" in the following and are labelled $C=\left\{c_{1}, \ldots, c_{k}\right\}$. Thus, children are assigned to placements within certain programs. We assume that each program decides on its own which children to accept, due to possibly divergent priority rankings. The fact that a program $c$ belongs to a facility $s$ is represented by $c \in s$. Analogously, we write $c \in S_{P r}$ and $c \in S_{P u b}$ to indicate how a program's preferences are expressed in the market mechanism.

Each of these programs has a placement capacity of $q_{c} \in Q$ of places that can be filled. In the following, we use different ranked lists. The preference rankings of children are labelled $P_{i}$ and the rank order preference lists of programs run by public providers are labelled $R_{c}$, respectively. The private providers play within each round manually and provide a ranked waiting list in each iteration $t$. The waiting lists are labelled $W_{c, t}$. In each iteration, these waiting lists can include an arbitrary number of children and are not limited by the capacity of the respective program.

Let $P_{i}$ be the preference ranking of a child $i$ concerning a subset of programs, such that $c \in P_{i}$ states that program $c$ is ranked by child $i$. The program $c$ is therefore strictly preferred by $i$ over remaining unassigned. Also, $c_{1} \succ_{P_{i}} c_{2}$ states that under preference ranking $P_{i}$ the child $i$ strictly prefers $c_{1}$ over $c_{2}$. Indifference between two programs is stated through $c_{1} \sim_{P_{i}} c_{2}$. The same applies to $R_{c}$ and $W_{c, t}$, although strict preference rankings are required here, without indifferences. During the assignment process, $\mu_{t}: I \rightarrow C \cup I$ denotes the current assignments at time $t$ in which every child is either assigned to a program or itself. The latter option means that the child is unassigned. Further, let the inverse of $\mu$ be defined as $\mu_{t}^{-1}(c)=\left\{i \mid \mu_{t}(i)=c\right\}$.

Since the private programs can adjust their waiting list in each iteration depending on their information about current matches, we use $\omega_{c}\left(\mu_{t}\right)=W_{c, t+1}$ to represent the waiting lists prior to the start of matching. Formally, we define 
our matching problem as $\phi\left(I, S_{P u b}, S_{P r}, Q, P_{I}, R_{C}, \omega_{C}\right)$. Since the first four parameters of $\phi$ are fixed for a given market from the perspective of the agents, in the following, we will simplify the problem definition as $\phi\left(P_{I}, R_{C}, \omega_{C}\right)$.

As a first step, parents submit the preference lists $P_{i}$ that rank the programs in tiers, whereby parents are indifferent between offers from the same tier. In addition to their preferred facilities, they can specify other preferences, such as the providers themselves, their opening hours, or the scope of care. In our mechanism, these formally stated preferences can be transformed into the weak preference lists $P_{i}$ over the set of programs $C$. In this way, a child could rank a placement at facility $A$ in a $45 \mathrm{~h}$ care program as preferable to a $45 \mathrm{~h}$ placement at facility $B$, which is in term preferable to a $35 \mathrm{~h}$ placement at facility $A$. The current iteration of manual offers is denoted by $t$, which is initialized with 1 . We further state that $\left|\mu_{0}^{-1}(c)\right|=0, \forall c \in C$.

The deferred acceptance mechanism with ties (DAT) is a multiple-round algorithm that assigns children in the following steps:

1. For every program $c \in S_{P r}$ : They receive a list of all feasible applicants. Feasible means that

a. $c \in P_{i}$ : The program is contained in the preference ranking of child $i$ and

b. $c \succ_{P_{i}} \mu_{t-1}(i)$ : Child $i$ strictly prefers program $c$ over the current match.

2. The decision-makers of program $c$ can select a set of children with size equal to $q_{c}-\left|\mu_{t-1}^{-1}(c)\right|$, which is the number of currently vacant placements, to send an offer to. Additionally, they can exceed their capacity and register as many children as they want on a strictly ranked priority list. The registered offers and the priority list together form the waiting list $W_{c, t}$.

3. Run program-proposing DA with the private programs' waiting lists $W_{c, t}$, the public programs' rank order lists $R_{c}$ and the children's preference lists $P_{i}$.

a. If $t \neq 1$ : Initialize the DA by assigning each child $i$ the place they held in $\mu_{t-1}$.

b. Private programs: Send out their registered offers (and subsequently offers based on $W_{c, t}$ ). 
c. Public programs: Send offers based on $R_{c}$.

d. Children: In each round of the DA, children hold the first offer for the highest tier according to $P_{i}$ and reject all others. Ties are broken at random (using either multiple or single tie-breaking).

e. For rejected offers: New offers for public and private programs' are submitted repeatedly based on their $R_{c}$ and $W_{c, t}$, respectively.

f. If no program makes any new offers, the resulting matching is called $\mu_{t}$.

4. After each DA run, the decision-makers of private programs can review all tier-one acceptances, deferred acceptances and rejections. The mechanism continues with the next round from step 1 .

5. This cycle terminates when a predefined number of rounds is exceeded, when no rejections are issued, or when no private facility registers a new offer.

\subsection{Game-theoretical Considerations}

Stability and strategy-proofness are typical performance desiderata considered in the theoretical literature on two-sided matching. In our context, stability is relevant for preventing justified envy, and, by extension, perceived unfairness, as specified in Requirement 1.1. Meanwhile, strategy-proofness is a necessary design element. We also have to consider the time to complete the matching process (see Requirement 2.3.2) and allow for complementarities in the form of preferences concerning group composition (see Requirement 4.2). In the following, we discuss the properties of the IDAT in view of these requirements.

The program-proposing DA is stable but not strategy-proof on the child's side (Roth \& Sotomayor, 1992). Roth (1982) generally shows that a DA cannot be stable and at the same time strategy-proof on both sides. Most practical applications implement the applicant-proposing version. The applicant-proposing DA is strategy-proof in theory. In practice, however, it is often subject to modifications that break strategy-proofness. Two of the most common modifications are to limit the number of programs to which a child may apply (see section 2.3) or to assign placements several times a year (e.g. Kennes et al., 2014). Also, the applicant-proposing DA is less suitable in our iterative context, as it 
allows programs to infer students' preferences based on the order in which proposals are received. This information induces strategic behaviour in applicants if programs consider this information when accepting proposals. We therefore consider the program-proposing DA and discuss its properties.

Azevedo \& Budish (2018) show that DA is "strategy-proof" in the large (SP-L). As the number of agents in a market grows, a mechanism is SP-L for children if for any $i \in I$ it is unlikely that there is a preference list $P_{i}^{\prime}$ such that $\phi\left(\left(P_{i}^{\prime}, P_{I_{-i}}\right), R_{C}, \omega_{C}\right) \succ_{P_{i}} \phi\left(P_{I}, R_{C}, \omega_{C}\right)$. In words, SP-L ensures that truthful reporting is approximately optimal for every agent. The childcare market at the municipal level can be considered large, and the proportion of agents who can successfully manipulate the mechanism should diminish in size when information (e.g. about capacities) is limited (Budish \& Cantillon, 2012). Further, Azevedo and Budish base these findings for DA being SP-L on the analysis of a weakening of envy-freeness by considering mechanisms that include any kind of tie-breaking lottery. They call this concept "envyfree but for tiebreaking." Their results state that a mechanism that includes tie-breaking can still be SP-L, provided no participant envies another simply due to a lower lottery number. Thus, our adaptation to allow for indifference within the children's preference lists, which is a form of tie-breaking, should not influence DAT being SP-L. In any case, successful manipulation requires broad information about the market, for which the probability diminishes if the market grows large.

Moreover, we argue that the potential loss in strategy-proofness of the program-proposing DA is tolerable in our scenario. From the children's side, the outcome can be manipulated through misrepresentation or truncation of the preference lists. Both will result in the realization of a different stable outcome, which is no longer program-optimal. Thus, manipulability is given if there are multiple stable outcomes in the market, which is observed to be unlikely in practice (Roth \& Peranson, 1999; Pathak \& Sönmez, 2008; Hitsch et al., 2010). In addition, the program side is bound to fixed admissions criteria (at least in the case of public providers), and thus cannot engage in strategic behavior to manipulate the market.

The DAT mechanism is stable, as the DA does not produce blocking pairs (Kojima \& Manea, 2010). Our iterative approach does not change this. Technically, a pair $(c, i)$ is a blocking pair for $\mu$ if

(i) $i$ and $c$ list each other in their ranking lists, 
(ii) $i$ is unassigned or prefers $c$ to $\mu(i)$, and

(iii) $c$ is undersubscribed or prefers $i$ to at least one member of $\mu(c)$.

Thus, there are no justified incentives for either applicant to leave their assigned matches. This also means that there is no justified envy between the applicants. When we allow for indifference on the applicant side, stability only holds if the applicants accept the strict preference rankings created through tiebreaking.

The presence of complementary preferences on the applicant side is widely studied in the context of couples in labor assignment problems (Roth \& Peranson, 1999; Kojima et al., 2013). In the market for childcare, siblings generate complementary preferences if parents want them to attend the same childcare facility together. Although complementarities on the program side have received little attention in the literature to date, they are particularly relevant to group composition. From a pedagogical point of view, it is generally accepted that programs aim to balance their intake in terms of age and gender. Aldershof \& Carducci (1996) show theoretically that in markets with complementary preferences, stable allocations are not guaranteed to exist. Annual intakes per program are often too small to add so-called slot-specific priorities, as suggested by Kominers \& Sönmez (2016). Research in the field of combinatorial auctions has shown that iterative mechanisms, in which offers can be made in rounds, allow participants to use a simple preference language for stating preferences regarding bundles (Parkes \& Ungar, 2000). Thus, the iterative approach of the DAT not only solves the challenges posed by provider autonomy (see Requirement 3.1), but also facilitates complementary preferences for group composition (see Requirement 4.2).

Nevertheless, the class of iterative DA mechanisms has been generally slow to converge (Bó \& Hakimov, 2016). Furthermore, any private facility has a clear incentive to delay the mechanism if it allows for complementary preferences. In particular, such a program would benefit from letting all other programs to move first, in order to choose freely from the remaining applicants in the last round (i.e. all applicants who would accept an offer from the program at that point). To speed up the mechanism, we induce facilities to send out offers fast by allowing for indifference tiers (weak preferences) on the applicant side. However, when combined with immediate acceptance, i.e. breaking weak preferences early, this results in efficiency losses (Erdil \& Ergin, 2008). 
We conclude that our modifications do in fact impair efficiency. Nonetheless, these losses are more than offset by the larger welfare gain of a faster process that ensures fairness. Overall, the proposed mechanism meets all desired performance requirements in the context of German childcare allocation.

\subsection{Information System}

To implement the revised mechanism in a real-life setting, we constructed the open-source matching platform Kitamatch. ${ }^{12}$ The software provides a comprehensive approach to the childcare matching problem, and is the first solution to make the decentralized deferred acceptance mechanism accessible (see Figure 3 for a screenshot).

The matching platform consists of four components: (i) a preference module for families, (ii) an administrative unit for municipal authorities, (iii) an interface for childcare facilities, and (iv) a matching mechanism unit. The software is designed to allow discrete use of a matching module and the importation of ranking lists from alternative software systems that register applications. $^{13}$

The municipality hosts the assignment process and is therefore also responsible for the software. It registers all public providers by their different programs, capacities, and criteria catalogs. In general, a criteria catalog assigns weights to each criterion in order to rank applicants objectively. For all public programs, this ranking $R_{c}$ is binding.

After registering on the website, families need to fill out a survey questionnaire about their preferred scope of care for their child $i$ and provide data relevant to the facilities' criteria catalogs. Most importantly, parents construct a tiered-based preference list $P_{i}$ via drag and drop over the programs $C$. From the perspective of the parents, each such program consists of the facility $s$ and the corresponding scope of care. The acceptance or rejection of potential offers is then fully automated. The applicant does not see temporary offers during the matching rounds and is only informed about his or her final allocation $\mu_{T}$ at the end of the matching process.

Each private childcare facility needs to register its different programs by

${ }_{12}$ The software is available under https://github.com/svengiegerich/ kitamatch.

${ }^{13}$ See for example Kitanavigator, https://www.itk-rheinland.de/, or KVJS, https://www. kitaweb-bw. de. 
name, capacity, and scope of care. Afterward, there is the option for every facility to create an individual criteria catalogue for the automatic presorting of applicants. As the matching process starts, each private program sees its feasible applicants presorted, and can add children to the waiting list $W_{c, t}$. After every round, a temporary matching $\mu_{t}$ is computed, and the software interface gives detailed feedback about acceptances and rejections. The programs can then decide once again to add applicants to the updated waiting list $W_{c, t+1}$. During every round, the waiting list can be shorter than the capacity of the program, it can exceed the capacity, or the facility can send no offer at all. This iterative process makes it possible to take group composition into account by conditioning offers in later rounds on previously formed matches.

The matching process takes several rounds. Within these rounds, private programs register offers manually to the waiting list $W_{c, t}$, while the preferences of children $P_{i}$ and public programs $R_{c}$, as they stick to their criteria catalogues, are automated. A round $t$ is closed either after a specific time interval (e.g. each day) or manually. The round-based computed matching $\mu_{t}$, as well as the rejected offers, are returned to the database. Afterwards, a new round starts. As soon as no private program sends any more offers, the matching process is considered complete, and the applicants are informed of the outcome $\mu_{T}$. The corresponding DAT mechanism is also available in the statistical $\mathrm{R}$ package matchingMarkets (Klein, 2021).

\section{EVALUATION}

To evaluate the proposed solution, we consider two aspects in this section. First, we document the implementation of our solution to provide evidence on how it resonates with stakeholders, including in particular childcare providers. Second, we provide simulation evidence on how well the mechanism scales for larger market sizes, different public/private provider shares, and different admissions criteria. This second aspect is relevant due to the number of rounds necessary for the mechanism to terminate and reach a stable matching. If the number of rounds grows too large, our approach would be too time-consuming and thus impractical. 


\subsection{Implementation}

We discuss the empirical results of our DAT implementation in the cities of Saerbeck and Greven. Comparing these two cities - which have divergent population sizes and preference profiles - yields particularly interesting results. To avoid repetition, when the results are similar or comparable, we portray our findings exclusively for Greven. Table 2 provides an overview of the results for both municipalities.

Greven, which has some 40,000 inhabitants, is a representative mid-sized city in Germany. The decentralized immediate acceptance (IA) process previously took four months to complete, and the matching repeatedly caused various problems (see section 2.1). Due to a new legal judgement (see Requirement 1.1), the city decided to reform its immediate acceptance (IA) process. The second city, Saerbeck, which has 7,000 inhabitants, used the same system and faced similar issues. In 2018, we were approached by the cities and asked to assist in redesigning their assignment systems. This request, in tandem with an ongoing case study in Münster, led to the development of the DAT mechanism. In the first implementation of the childcare year 2019/20, the mechanism was implemented as described in section 4.1, without the feature of indifference tiers. The following analysis, therefore, is silent on the effects of this feature. The simulations in section 5.2, however, shed some light on this issue.

The Greven childcare setting consisted of 479 children and 26 childcare facilities, including one public facility. The facilities differentiated their care offers between the age cohorts $U 2$ (under two years old), 2 (two years old) and $O 3$ (older than three years), which resulted in 65 programs. All childcare facilities constructed point-based criteria catalogs that assigned points to rank applicants. These catalogs were quite heterogeneous between facilities, both with a view to the criteria used and their number, which varied between three and eight. Without exception, all of the facilities used common criteria regarding siblings and the employment. In addition, some facilities include the denomination of the child, and others consider more abstract criteria such as family emergency (illness), inclusion, and cultural diversity. Saerbeck's institutions use a similar range of criteria, but apply the criteria in strictly lexicographical order.

In both municipalities, children's preferences were collected using a written questionnaire. On average, parents indicated 3.29 programs in Greven and 


\begin{tabular}{lcccc}
\hline Market & \# Children & \# Programs & \# Providers & Avg.Preference Length \\
\hline Greven & 479 & 65 & 26 & 3.29 \\
Saerbeck & 100 & 18 & 7 & 2.73 \\
\hline
\end{tabular}

Table 1: Greven's and Saerbeck's childcare assignment setting.

2.73 in Saerbeck in their preference list.

For the actual matching process in Greven, all heads of the childcare institutions were invited to the youth welfare office in January 2020. The matching in Saerbeck took place in January 2019. In both meetings, participants were presented with a pre-sorted list of applicants based on points constructed by their submitted criteria catalogs. The sorting only served as a suggestion; facilities were allowed to consider group complementarities as they sent out decentralized offers through the matching platform round by round. Since all decision-makers sat together, it was possible to conduct the matching rounds flexibly instead of waiting for fixed time intervals. The final matching in Greven and Saerbeck took an hour and was completed in seven and six rounds, respectively. In total, 403 of 479 and 85 of 100 children, respectively, were assigned a place. Thus, none of the markets cleared their full capacity. This can be mainly explained by two factors. First, many parents indicated only a few - in $9.6 \%$ (31\%) of the cases just one - acceptable childcare programs. Second, parents had no information beforehand on which programs had available placements. In fact, $19 \%$ (24\%) of all parents listed programs that did not have a single vacant care place. Some preference lists even shared both of these problems simultaneously. Thus, these children's preference lists were practically empty.

\subsubsection{Welfare analysis}

This section concludes with a welfare analysis that constructs counterfactuals for the matches in Greven and Saerbeck to compare the performance of three mechanisms: (i) the previously used IA, (ii) the standard program-proposing DA, and (iii) the DAT, which allows facilities to deviate from the pre-sorted priority list. This comparison is possible because we observe for all childcare facilities both (i) their ranking of applicants based on a criteria catalogue, denoted by $r_{\text {catalogue }}$, and (ii) their revealed preferences from the sequence of 


\begin{tabular}{|c|c|c|c|c|c|}
\hline \multirow{2}{*}{ Mechanism } & \multirow{2}{*}{ \# Matches } & \multirow{2}{*}{$\begin{array}{l}\text { Children's } \\
\text { avg. rank }\end{array}$} & \multicolumn{2}{|c|}{ \# Blocking pairs ${ }^{*}$} & \multirow{2}{*}{$\begin{array}{c}\text { Group } \\
\text { complements }\end{array}$} \\
\hline & & & with $r_{\text {catalogue }}$ & with $r_{\text {revealed }}$ & \\
\hline \multicolumn{6}{|l|}{ Greven } \\
\hline IA & 401 & 1.15 & 84 & 90 & No \\
\hline DA & 406 & 1.34 & 0 & 19 & No \\
\hline DAT & 403 & 1.33 & 54 & 0 & Yes \\
\hline \multicolumn{6}{|l|}{ Saerbeck } \\
\hline IA & 86 & 1.31 & 14 & - & No \\
\hline DA & 85 & 1.36 & 0 & - & No \\
\hline DAT & 85 & 1.33 & - & 0 & Yes \\
\hline
\end{tabular}

Table 2: Comparison of the redesigned DAT with simulated results for immediate acceptance (IA) and deferred acceptance (DA) algorithms in the cities of Greven and Saerbeck. The DAT respects group complementarities as it allows round-based deviations from the preference list - used in 12\% (9\%) of all sent offers made in Greven (Saerbeck) from round two onwards.

$\left(^{*}\right)$ Blocking pairs are evaluated based on the ranking of facilities using both the criteria catalogue ( $\left.r_{\text {catalogue }}\right)$ and facilities' revealed preferences $\left(r_{\text {revealed }}\right)$. The latter could not be calculated for Saerbeck because of missing information.

offers made in the DAT, denoted by $r_{\text {revealed }}$. In what follows, we analyze four aspects: unfilled placements; applicant preferences; facility preferences and participation constraints; and fairness.

A first observation from Table 2 is that no mechanism allocates the full number of placements available. ${ }^{14}$ In Greven (Saerbeck), the IA assigns 401 (86) children, the DA 406 (85) and the DAT 403 out of 469 (85 out of 101) available places. The places are free primarily due to the incomplete ranking lists of the parents.

The average rank of the assigned placements based on parent preferences is relatively equal across all mechanisms in both cities. The IA allocates more extreme matches, as more children get their first or their last rank, while the DA and DAT both result in a more balanced distribution.

For an analysis of facility preferences, we consider their ranking over applicants as inferred from the sequence of offers made, $r_{\text {revealed }}$. This measure is more appropriate than the ranking generated from the criteria catalog, because

${ }^{14}$ For the city of Greven, the available placements were determined as reported in Table 4. 
it also captures deviations in the DAT that allow facilities to express richer preferences and, in particular, to account for complementarities. Group complementarities were of importance for programs, as they deviated from their priorities several times during the matching process. For example, in Saerbeck, a program with three placements sent an offer to the girl ranked fourth in round two, as it already held two boys from round one. As this girl rejected the offer, the facility skipped the following boys with rank five and six and instead sent an offer in subsequent rounds to the girls in seventh and eighth place. Thus, the program made a trade-off between priorities and group composition. Overall, in Greven (Saerbeck), 12 out of 26 programs (8 out of 18) deviated from the pre-sorted prioritization at least once. In total, we find a deviation rate from the pre-sorted rankings of $12 \%(9 \%)$ when considering all rounds, starting from round two to the final round seven. See Table 3 in the Appendix for an overview of results for Greven. We observe that in Greven, in $32 \%$ of all deviations, applicants were preferred because they added heterogeneity in the gender composition and in $15 \%$ due to heterogeneity in age. ${ }^{15}$

We measure the welfare effects enjoyed by facilities when using DAT rather than DA in terms of the number of programs that would block the implementation of DA matching. In particular, we know that no program would oppose the implementation of DA matching if $r_{\text {catalogue }}$ is identical to $r_{\text {revealed }}$. That is, if programs' revealed preferences in $r_{\text {revealed }}$ do not deviate from the critera catalog in $r_{\text {catalogue }}$. If programs, however, have complementary preferences, then these will be expressed in terms of deviation from the criteria catalog, and result in blocking pairs. Using $r_{\text {revealed }}$, we find for Greven a total of 19 blocking pairs in the DA matching. These 19 blocking pairs involve 13 programs and 14 children. This means that 13 out of the 65 programs would block the implementation of DA matching, and would presumably only agree to participate in the matching scheme if the DAT were used instead. ${ }^{16}$

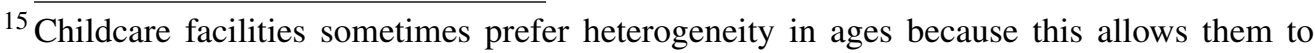
distribute the settling-in periods for children evenly over different years, rather than having a high turnover in any particular year.

${ }^{16}$ In this welfare analysis for facilities, we do not consider the alternative welfare measure of the average assigned rank (as we did for the analysis of applicant preferences in Table 2, column 3). This is because of the following complication. In the DAT, programs that strictly follow ranking $r_{\text {catalogue }}$ in the mechanism, can have a sequence of offers $r_{\text {revealed }}$ that is a shortened version of $r_{\text {catalogue }}$. This is because some children in $r_{\text {catalogue }}$ cannot be selected by the program in the mechanism if the children already hold their first preference from another program. This complication, however, does not affect our blocking pair measure, since offers 
We now turn to the welfare analysis for applicants. As seen in the analysis of facility preferences, the DAT allows facilities to deviate from the criteria catalogue, which may be a crucial feature for ensuring all facilities participate on a voluntary basis. For applicants, however, the DAT can be perceived as unfair, when $r_{\text {revealed }}$ is not identical to $r_{\text {catalogue }}$. In this case, applicants will have justified envy of other applicants. The magnitude of this problem can be measured by asking how many applicants would block the DAT matching. Using $r_{\text {catalogue }}$, we find for the city of Greven a total of 54 blocking pairs in the DAT. These 54 blocking pairs involve 13 programs and 49 children. This means that 49 out of the 403 matched applicants would oppose the DAT matching. While this number is still lower than the total of 77 blocking pairs we find when applying the previously-used IA mechanism for Greven (respectively 14 in Saerbeck), ${ }^{17}$ it shows that fairness may have to be compromised, even in the DAT, in order to respect provider autonomy and achieve voluntary participation.

\subsection{Scalability}

We simulate childcare markets and evaluate the effect of different parameter settings on the required number of iterations. ${ }^{18}$ All settings are based on the childcare market in the city of Münster, which had a total of 3,031 childcare placements in 2020 (Press and Information Office of the City of Münster, 2019). Although these placements are separated between children younger than three and older than three, there are 2,810 children below the age of three in need of a place. As in the baseline scenario, we therefore use a market consisting of 3000 applicants and 200 facilities. Each facility has, on average, three programs, resulting in a total of 600 programs. We work with 1.2 applicants per childcare place, which reflects the shortage of placements for children under the age of 3 in many German cities. ${ }^{19}$ Finally, we randomize

to these children would have been rejected anyway.

${ }^{17}$ Thus, in the old procedure around $14 \%$ to $20 \%$ of all children were disadvantaged because of the unstable process.

18 The code to reproduce all simulations and figures can be found on: https://github . com/tobiasreischmann/matchingmarkets-simulation

${ }^{19}$ In Germany, $43 \%$ of the parents with children aged below three apply for a childcare placement, but only $35 \%$ can be offered one, leading demand to outstrip supply by a ratio of 1.23:1. (For more see, https://www.kindergartenpaedagogik.de/ fachartikel/kita-politik/bildungspolitik/1650) 
programs to be private facilities with probability $q$, which we refer to as the private facility share.

The simulated preferences of applicants and programs combine horizontal, vertical, and idiosyncratic components, ${ }^{20}$ and are modeled using two selection functions: one for the applicant-side vis-à-vis the program-side, and vice versa. The selection functions operate on randomly generated attributes assigned to applicants and programs. These attributes are then used to calculate and combine the three preference components into a lexicographic preference function.

\subsubsection{Applicant preferences}

For horizontal preferences, $x$ and $y$ coordinates are assigned to applicants and programs as geographical information. Applicants are modeled to be indifferent about programs within the same city district if the decision is solely based on distance. Thus, $x$ and $y$ are realizations from a uniformly distributed categorical variable from 1 to $i$. $i$ is chosen depending on the number of programs, such that there are about ten programs within the same district. Horizontal preferences are then determined using the Euclidean distance.

Vertical preferences are modeled by a variable that assigns a quality level to each program, which is used directly in the applicants' selection function.

The idiosyncratic component of applicants' preferences assigns each applicant and each program one out of ten random types. Applicants only value a program if it is of the same type. This accounts for valuation based on religious beliefs or special forms of care.

In addition to the three preference components, each applicant receives three uniformly distributed variables that determine which of the three preference aspects carries highest importance for the respective applicant.

Finally, we include the effects of allowed indifference within the children's ranking lists in our simulation. In general, each child only ranks a small subset of the whole market. In the baseline scenario of 600 programs, each child ranks 30 of them. If indifferences are allowed, the ranking lists of the children are

${ }^{20}$ Applicant preferences are defined as follows. A horizontal component captures applicants' preferences for attributes of programs that are closer to their own attributes, such as the geographical distance between the applicant and program. The vertical component captures program attributes that all applicants value equally, such as a high staff ratio. Finally, the idiosyncratic component models subjective valuations. 
separated into a set of predefined tiers of equal size. In the baseline scenario, these are four tiers sized three, seven, ten and ten. For other market sizes, the size of the ranking lists and the tiers are adjusted accordingly.

\subsubsection{Program preferences}

A similar selection function is used on the program side. The design of this function is guided by the admissions criteria of the city of Münster, which requires providers to consider the three preference components in a strict order.

For the horizontal component, each program only values applicants from the program's home district. Actual distance does not matter. For the vertical component, applicants are assigned a priority variable, which represents the family's social need or the age of the child.

The idiosyncratic component is also generated randomly for each combination of applicant and program. It models the subjective valuations of the program staff. This component only enters into the selection function of private providers. For public providers, the inclusion of subjective factors is ruled out by law.

The preference rankings of the programs are usually model criteria catalogues, which have a strict order of vertical and horizontal components. For the preference generation, we use two random variables, which provide the three preference components in a strict order for each program.

\subsubsection{Simulation}

In our analysis, we evaluate how different market characteristics impact the number of rounds required in the DAT. To this end, we simulate several markets under different parameter settings and obtain the necessary number of rounds until at least $95 \%$ of the matches in the final DAT results are reached.

Figure 1 shows how the assigned placements change during the matching process in the baseline scenario described above. In each iteration, we differentiate three assignment states. For unmatched placements, no assignment exists in the current iteration. While final assignments represent a match that is also part of the final iteration's match, temporary assignments are changed in future iterations. Although most of the placements quickly reach their final allocation, some placements are frequently changed, which causes a significant number of rounds, if we wish to run the process to its natural end. However, we argue that ending a matching process early when the percentage of non-final assignments 


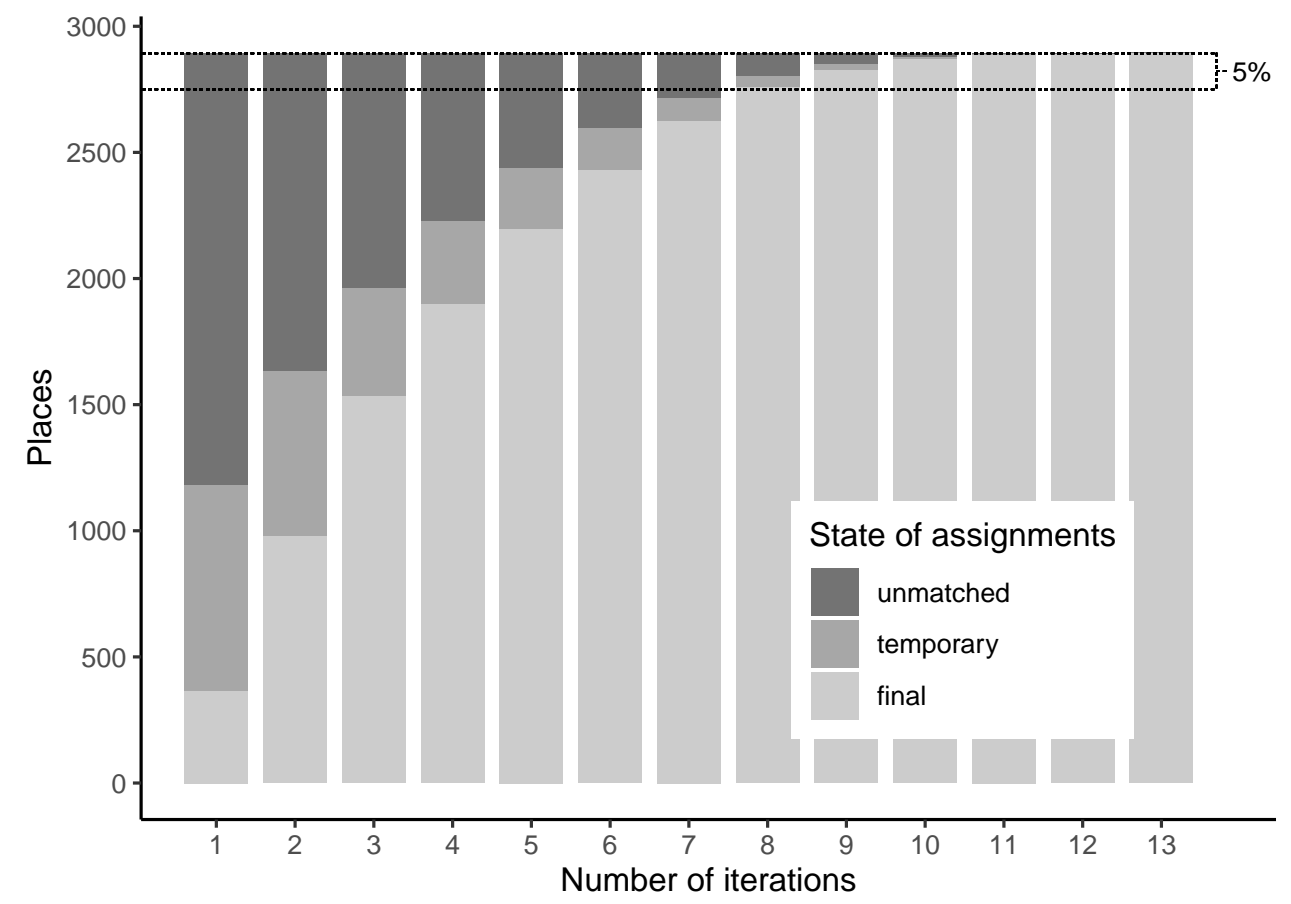

Figure 1: Matching after $t$ iterations in comparison to a full DAT run (with 60\% private facilities, occupancy rate of 1.2, 3000 applicants, and 600 programs)

is below 5\% is tolerable given the advantage this brings in making the mechanism more practical. In practice, the admission criteria have to ensure that no hardship cases are among the 5\% (or among the even smaller number of unmatched assignments). Since the hardship cases are usually ranked higher and thus receive offers earlier, it is unlikely that hardship cases will fail to receive offers within the first couple of rounds.

Figure 2 illustrates how different parameters affect the number of required iterations. For this evaluation, we build an average of played rounds for ten simulated markets. The evaluated characteristics are:

1. Occupancy rate (number of children over the number of available placements), ranging from 0.2 to 3. Baseline scenario: 1.2 .

2. Share of private facilities, ranging from $q=0 \%$ to $100 \%$. Baseline scenario: 0.8 . 
3. Applicants can rank facilities in tiers (yes/no). Baseline scenario: yes.

4. Number of applicants (keeping the number of programs and the occupancy rate fixed), ranging from 600 to 5,000. Baseline scenario: 3, 000 .

5. Length of children's ranking lists. Baseline scenario: 30 separated into four tiers of 3,7,10, and 10 programs.

6. Threshold for the percentage of the stable matches to be reached, ranging from $93 \%$ to $100 \%$. Baseline scenario: $95 \%$.

7. Market size, ranging from 250 applicants and 50 programs to 6,000 and 1,200. Baseline scenario: 3,000 and 600.

8. Selection function of the programs, including categories "mixed preferences"; "vertical only"; "horizontal only"; "both sides vertical only". Baseline scenario: mixed preferences.

Our motivation for inclusion of the last characteristic is the controversial discussion in the city of Münster as to whether children should receive priority at a facility if they live in the same district. In addition, this final evaluation serves as a proof of concept for our simulation. We represent this through the inclusion of different combinations of preference aspects within the selection functions. While mixed preferences are a feature of the scenario described above, the next three scenarios restrict the programs' preferences to only one aspect. The last scenario assumes that both the children and the facilities only care about vertical preferences. This scenario represents an edge case, which leads logically to a significant increase in necessary rounds.

Within the eight analyzed characteristics, each city has scope for decisionmaking. While the characteristics 1, 2, 4 and 7 can be assumed to be fixed within a given market, each city can freely choose their ranking strategies for children, the applied threshold for the mechanism, and the selection function of the programs. They can also influence the number of programs a child can rank via the implemented admissions system. Additionally, a city can strive to convince childcare providers to provide full ranking lists through objective admission criteria, thus reducing the private facility share. Also, in the long run, the cities can provide more placements for the children, which will change the occupancy rate. Moreover, the number of rounds that are deemed applicable depends on the way the rounds are played. If it is possible 
to place all decision-makers into one room and play all rounds in one day, a higher number of rounds might be tolerable. If the childcare providers agree on playing one round each week, each additional round will delay the time when the parents are informed about the final assignments. We argue that a suitable number of rounds might be around six to nine. This area is highlighted in Figure 2 through the grey areas.

The results show that neither the occupancy rate (Figure 2.1) nor the number of applicants (Figure 2.5) has a significant influence on the number of rounds played. With Figure 2.5, the number of placements was scaled, since the occupancy rate and the number of providers stayed fixed. At the same time, the number of rounds played grows linear with a higher private facility share (Figure 2.2) and a lower threshold (Figure 2.4). However, we can conclude that a lower threshold and thus a higher convergence to the stable matching might be applicable in cities with a lower share of private facilities. Also, letting the children rate facilities in tiers (Figure 2.3) has a positive impact on the number of rounds played. The reason is that longer preference lists on the children's side of the market will cause a higher number of rounds. With tiers, the preference lists are shortened more rapidly during the matching process.

As mentioned, the length of the children's ranking lists has a significant effect on the number of rounds played (Figure 2.6). In practice, children are highly unlikely to rank the full market. According to the survey, the average of facilities ranked was 3.4, which does not even exhaust the list of seven facilities for which parents were allowed to apply.

Figure 2.7 reveals the impact of the market size on the number of rounds played. While the number of rounds increases with the size of the market, the number of rounds generally remains in a manageable range.

Finally, Figure 2.8 highlights two intriguing results. First, the actual admissions criteria that the facilities apply seem to have almost no effect on the number of rounds played. Thus, the facilities do not have to restrict their method of ranking children for the mechanism to be viable in practice. Furthermore, we also find in Figure 2.8 evidence for the hypothesis that the mechanism will break if the preferences are solely vertical. In theory, this would cause as many rounds as the number of placements a facility has. In our case, this effect is limited due to the short preference lists of the children. Through our survey, we expect that the distance to a facility plays a crucial role in the decision-making of the parents. ${ }^{21}$ Thus, a scenario in which both

${ }^{21}$ In a survey, parents were asked about the characteristics that influence their preference rankings. 


\section{1: Occupancy Rate}

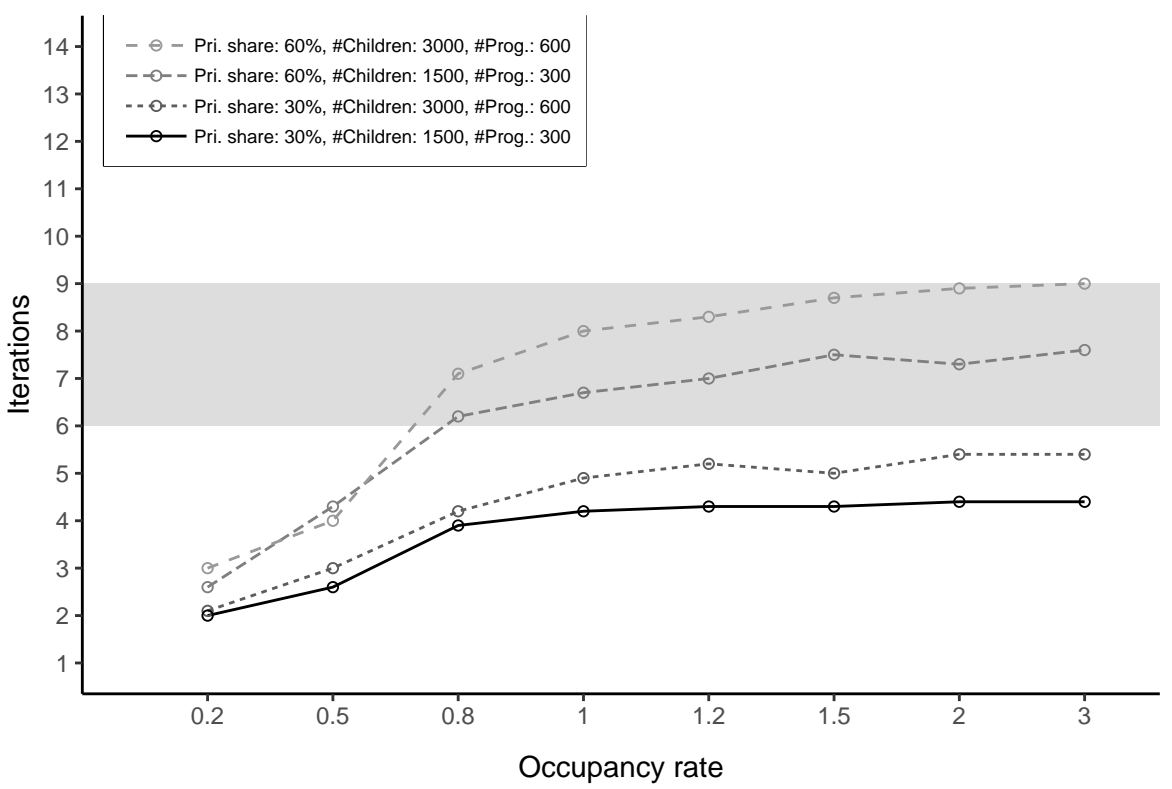

2.2: Share of private facilities

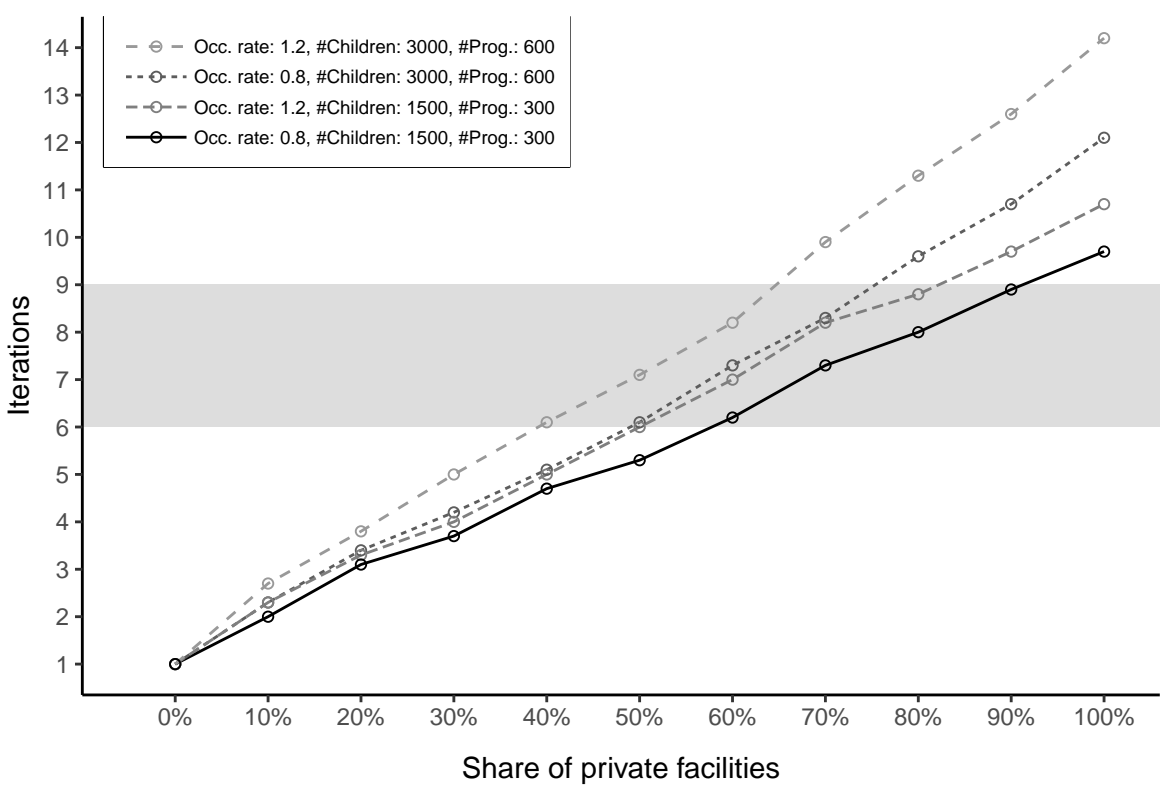

Figure 2: Effects of different parameters on the number of iterations. 


\section{3: Tiers, with or without}

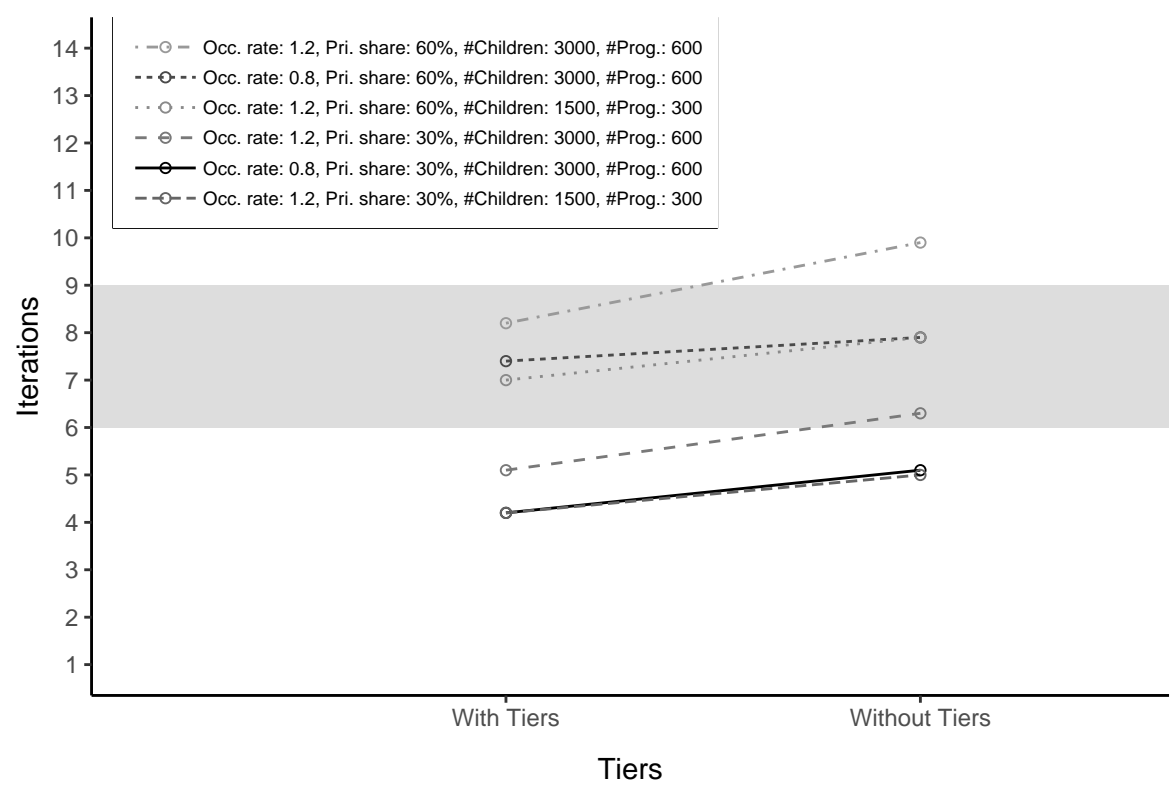

\section{4: Threshold}

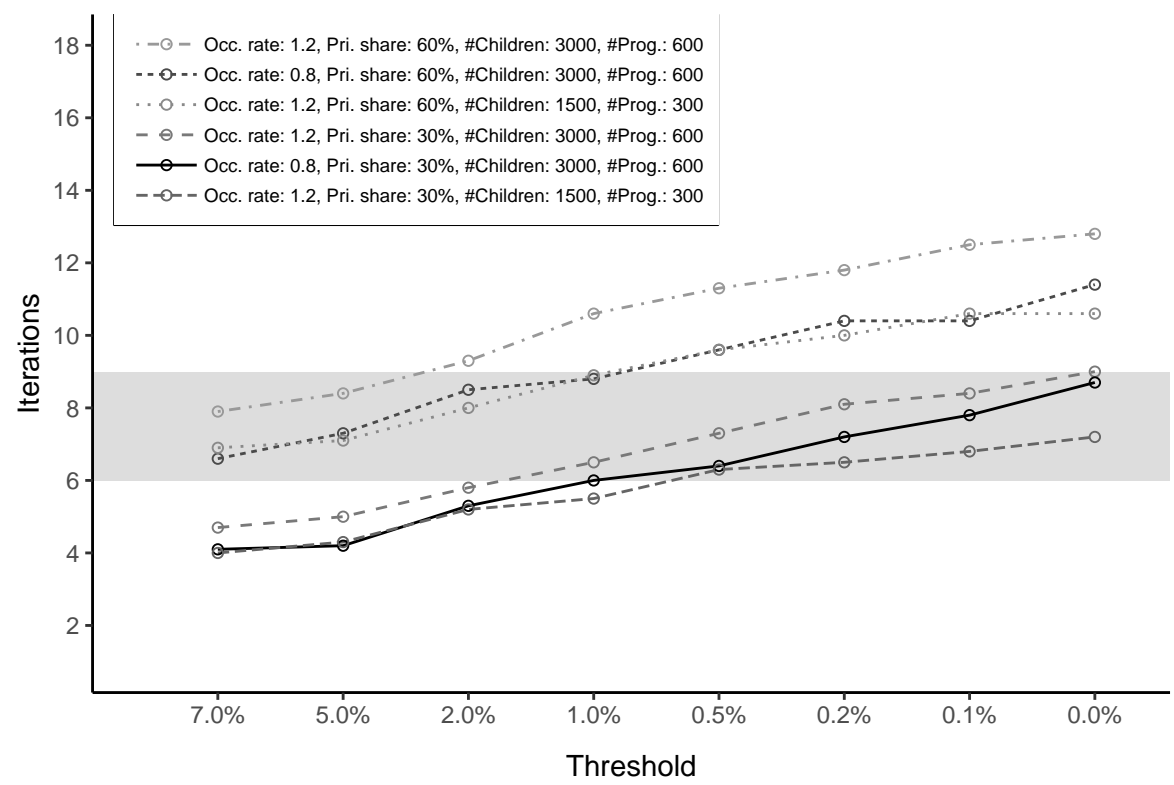

Figure 2: Effects of different parameters on the number of iterations (cont.). 


\section{5: Number of applicants}

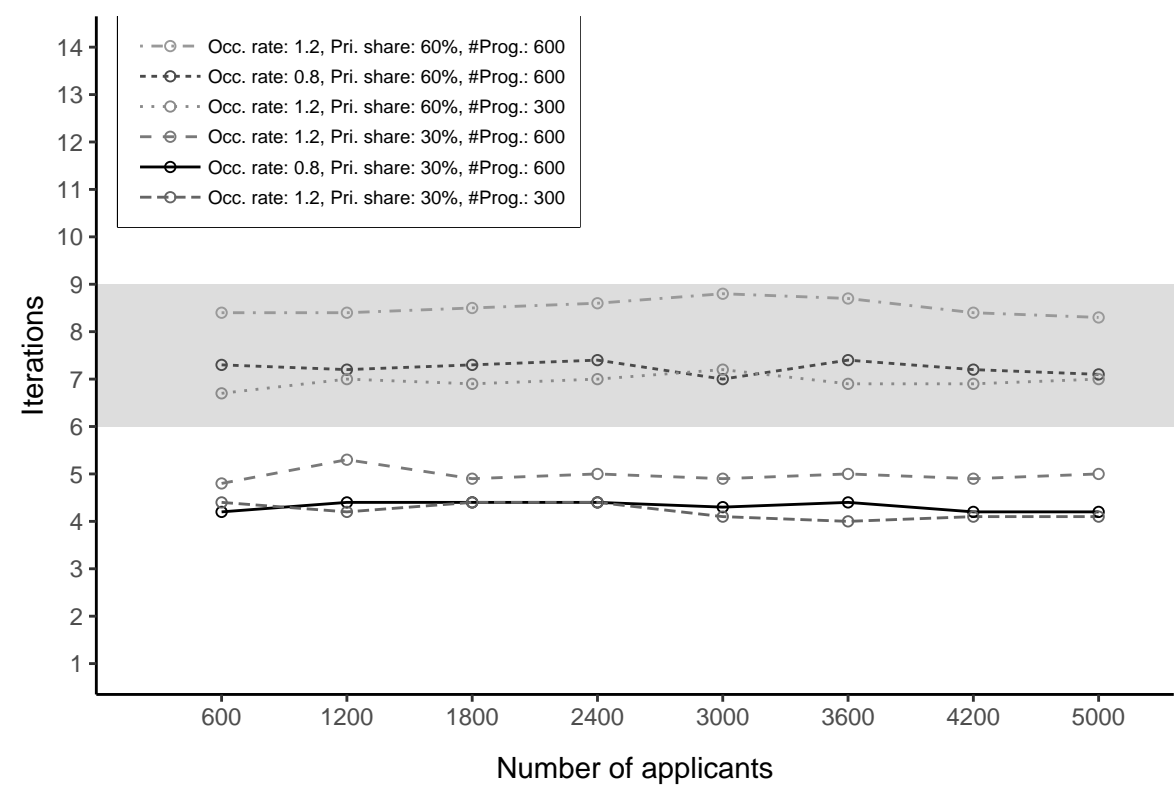

2.6: Length of ranking list

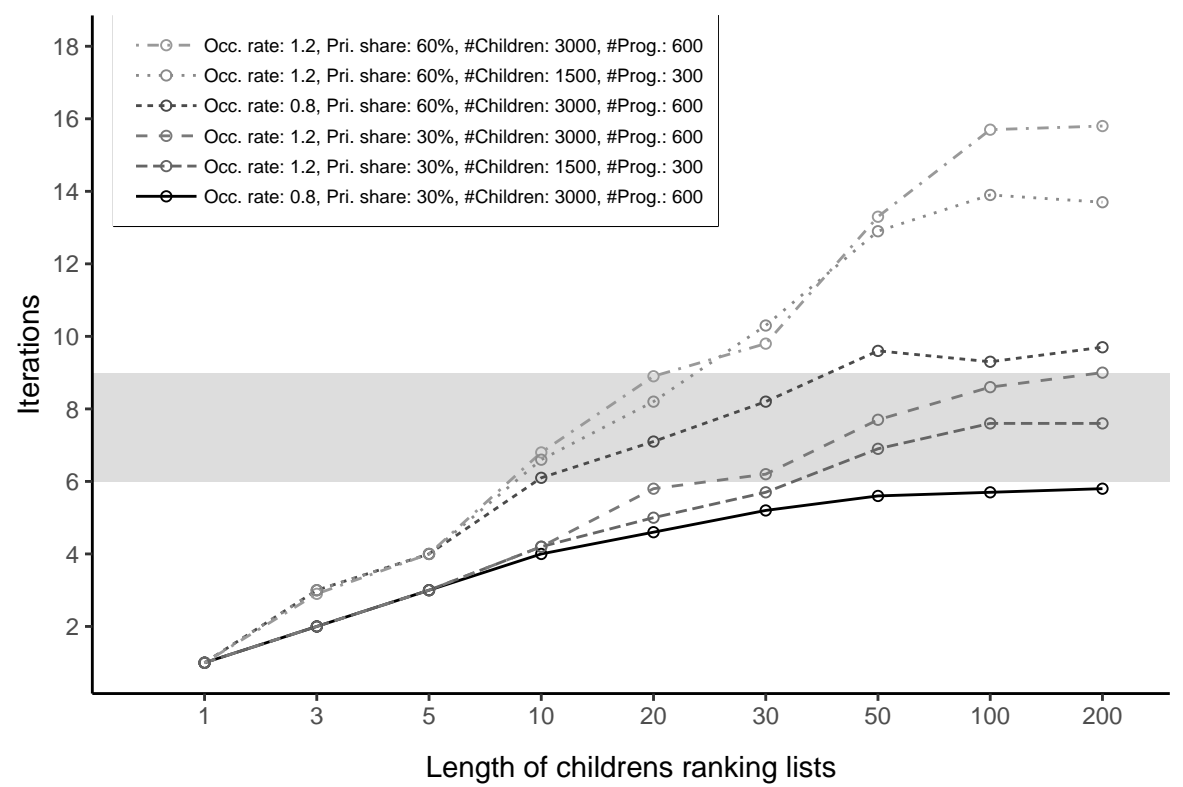

Figure 2: Effects of different parameters on the number of iterations (cont.). 


\section{7: Market size}

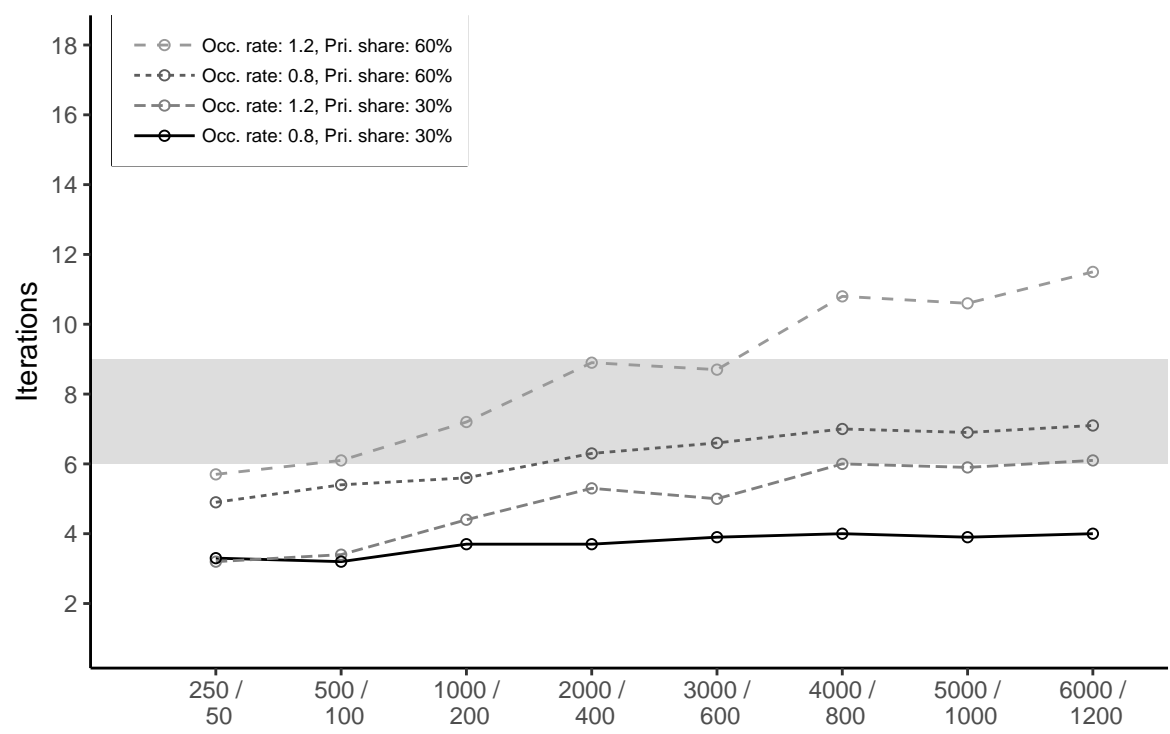

Market size (no. of applications / no. of programmes)

\section{8: Preference scenarios}

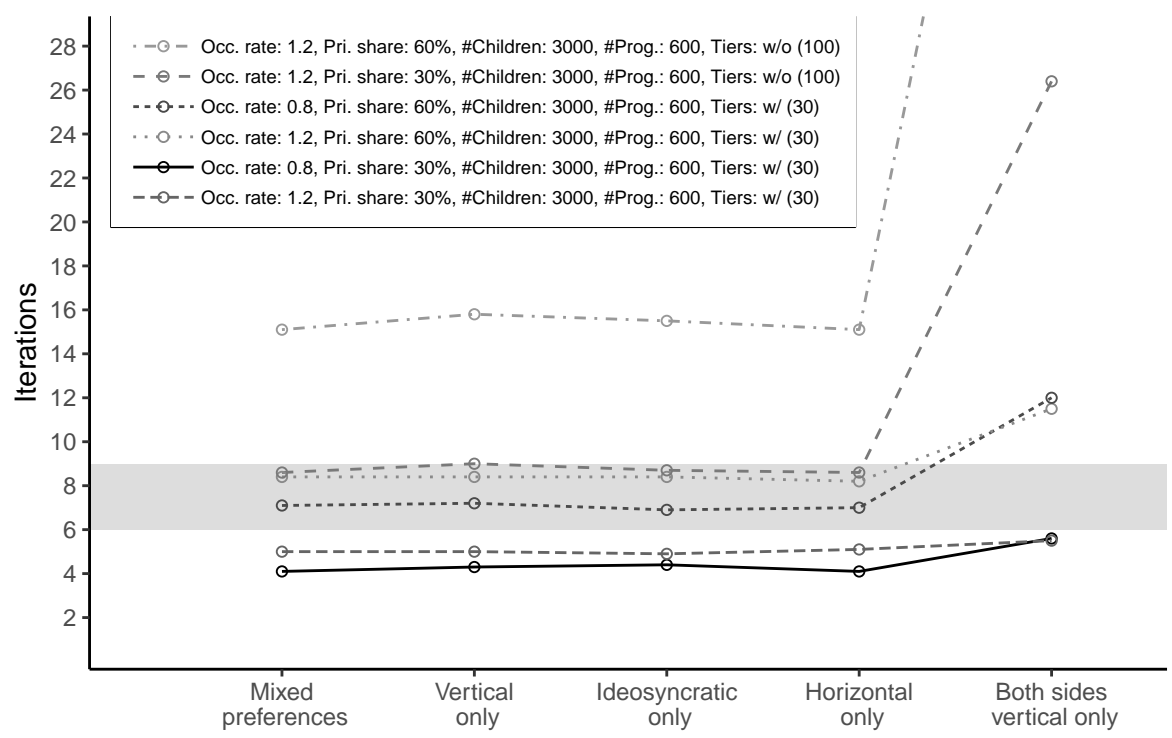

Preference Scenario

Figure 2: Effects of different parameters on the number of iterations (cont.). 
sides of the market only exhibit vertical preferences seems unlikely.

\section{CONCLUSION}

In this paper, we analyzed the challenges attendant to a redesign of the childcare market in German cities, including in particular the requirements that the allocation mechanism needs to fulfill. In a case study, we identified fairness and speed as two significant issues in current allocation practice. While the matching literature has successfully addressed various matching problems, it is not perfectly applicable to the German childcare market, for the reasons discussed in the foregoing. To solve these issues, we introduced a deferred acceptance mechanism with ties (DAT), which is tailored to the challenges we identified and which ensures a decentralized, fast, and fair allocation process.

The applicability of the new mechanism was also tested in two German cities. Furthermore, we performed a simulation of the mechanism in different market settings, showing which market characteristics affect its performance. We find that while the new mechanism appears to be applicable to various markets, it has to be adjusted to specific market needs.

Although our solution certainly solves problems shared by many German cities, other cities might have additional requirements that need to be incorporated into the mechanism's design. One such requirement is moving from an annual to a monthly allocation schedule, which adds strategic issues if parents postpone their applications to secure a better placement. In the end, the successful implementation of a redesigned mechanism is dependent on local policy and stakeholder support for reform. Further cooperative activities with other cities for the future implementation of the mechanism are already underway.

They could select from a set of eight preselected characteristics (e.g. facility distance, quality, opening hours) and were asked to provide a ranking for them. The parents could indicate a maximum of 6 characteristics and were also able to provide other characteristics using a free text field. The distance from home was selected by $79 \%$ of all parents as a relevant characteristic. Also, $50 \%$ of all parents ranked it at first or second place in $50 \%$ of the cases. Distance to work and trip to work scored significantly lower, with only $23 \%$ and $35 \%$ of parents mentioning it as an influencing characteristic. 


\section{References}

Ágoston, K. C., Biró, P., \& Szántó, R. (2018). Stable project allocation under distributional constraints. Operations Research Perspectives, 5, 59-68.

Aldershof, B., \& Carducci, O. M. (1996). Stable matchings with couples. Discrete Applied Mathematics, 68(1-2), 203-207.

Aygün, O., \& Turhan, B. (2020). Dynamic reserves in matching markets. Journal of Economic Theory, 105069.

Azevedo, E. M., \& Budish, E. (2018). Strategy-proofness in the large. Review of Economic Studies, 86(1), 81-116.

Biró, P. (2017). Applications of matching models under preferences. In U. Endriss (Ed.), Trends in Computational Social Choice. COST: European Cooperation in Science and Technology.

Bó, I., \& Hakimov, R. (2016). Iterative versus standard deferred acceptance: Experimental evidence. Working paper, SP II 2016-209.

Braun, S., Dwenger, N., \& Kübler, D. (2010). Telling the truth may not pay off: An empirical study of centralized university admissions in Germany. BE Journal of Economic Analysis \& Policy, 10(1).

Budish, E., \& Cantillon, E. (2012). The multi-unit assignment problem: Theory and evidence from course allocation at Harvard. American Economic Review, 102(5), 2237-71.

Bös, N. (2017). Raus aus der Kita-Warteschlange. Frankfurter Allgemeine Zeitung. Retrieved 2021-10-10, from https://www.faz.net/ aktuell/wirtschaft/kinderbetreuung-raus-aus-der-kita -warteschlange-15053793.html

Carlsson, S., \& Thomsen, S. (2014). Nicht ausgeschöpfte Potenziale in der KitaPlatzvergabe. Vierteljahrshefte zur Wirtschaftsforschung, 83(1), 183-198.

Chen, Y., \& Kesten, O. (2017). Chinese college admissions and school choice reforms: A theoretical analysis. Journal of Political Economy, 125(1), 99-139.

Delacrétaz, D., Kominers, S. D., \& Teytelboym, A. (2016). Refugee resettlement. Working paper.

Drummond, J., Perrault, A., \& Bacchus, F. (2015). SAT is an effective and complete method for solving stable matching problems with couples. Twenty-Fourth International Joint Conference on Artificial Intelligence.

Echenique, F., Wilson, A. J., \& Yariv, L. (2016). Clearinghouses for two-sided matching: An experimental study. Quantitative Economics, 7(2), 449-482.

Erdil, A., \& Ergin, H. (2008). What's the matter with tie-breaking? Improving efficiency in school choice. American Economic Review, 98(3), 669-89.

Erdil, A., \& Ergin, H. (2017). Two-sided matching with indifferences. Journal of 
Economic Theory, 171, 268-292.

Gehlke, A., Hachmeister, C.-D., Hüning, L., \& de Vries, L. (2017). Der CHE Numerus Clausus-Check 2017/18. Eine Analyse des Anteils von NC-Studiengängen in den einzelnen Bundesländern. CHE Centre for Higher Education.

Geitle, A., Johnsen, Ø., Ruud, H., Fagerholt, K., \& Julsvoll, C. (2020). Kindergarten allocation in Norway: An integer programming approach. Journal of the Operational Research Society, 1-10.

Gonczarowski, Y. A., Nisan, N., Kovalio, L., \& Romm, A. (2019). Matching for the Israeli "Mechinot" gap-year programs: Handling rich diversity requirements. Proceedings of the 2019 ACM Conference on Economics and Computation, 321321.

Gong, B., \& Liang, Y. (2017). A dynamic college admission mechanism in Inner Mongolia: Theory and experiment. Working paper.

Grenet, J., He, Y., \& Kübler, D. (2019). Decentralizing centralized matching markets: Implications from early offers in university admissions. WZB Discussion Paper.

Hafalir, I. E., Yenmez, M. B., \& Yildirim, M. A. (2013). Effective affirmative action in school choice. Theoretical Economics, 8(2), 325-363.

Hatfield, J. W., \& Milgrom, P. R. (2005). Matching with contracts. American Economic Review, 95(4), 913-935.

Hitsch, G. J., Hortaçsu, A., \& Ariely, D. (2010). Matching and sorting in online dating. American Economic Review, 100(1), 130-63.

Kamada, Y., \& Kojima, F. (2018). Stability and strategy-proofness for matching with constraints: A necessary and sufficient condition. Theoretical Economics, 13(2), 761-793.

Kamada, Y., \& Kojima, F. (2020). Fair matching under constraints: Theory and applications. Working paper.

Kennes, J., Monte, D., \& Tumennasan, N. (2014). The day care assignment: A dynamic matching problem. American Economic Journal: Microeconomics, 6(4), 362-406.

Klein, T. (2021). Analysis of stable matchings in R: Package matchingMarkets. Vignette to $\mathrm{R}$ package matchingMarkets. The Comprehensive R Archive Network.

Klein, T., \& Herzog, S. (2018). Matching practices for childcare in Germany. Retrieved 2021-10-10, from http: / /www. matching-in-practice.eu/ matching-practices-for-childcare-germany/

Klijn, F., Pais, J., \& Vorsatz, M. (2019). Static versus dynamic deferred acceptance in school choice: Theory and experiment. Games and Economic Behavior, 113, 147-163.

Kojima, F. (2012). School choice: Impossibilities for affirmative action. Games and Economic Behavior, 75(2), 685-693. 
Kojima, F., \& Manea, M. (2010). Axioms for deferred acceptance. Econometrica, 78(2), 633-653.

Kojima, F., Pathak, P. A., \& Roth, A. E. (2013). Matching with couples: Stability and incentives in large markets. Quarterly Journal of Economics, 128(4), 1585-1632.

Kominers, S. D., \& Sönmez, T. (2016). Matching with slot-specific priorities: Theory. Theoretical Economics, 11(2), 683-710.

Konegen-Grenier, C. (2018). Wer bekommt einen Studienplatz? Die Regelung des Hochschulzugangs im Umbruch. IW-Report.

Mennle, T., \& Seuken, S. (2017). Trade-offs in school choice: Comparing deferred acceptance, the classic and the adaptive Boston mechanism. Working paper.

Nguyen, T., \& Vohra, R. (2019). Stable matching with proportionality constraints. Operations Research, 67(6), 1503-1519.

OVG NRW. (2017). Entscheidung des Oberverwaltungsgericht Nordrhein-Westfalen, 12 B 930/17.

Parkes, D. C., \& Ungar, L. H. (2000). Iterative combinatorial auctions: Theory and practice. 17th National Conference on Artificial Intelligence, 74-81.

Pathak, P. A., \& Sönmez, T. (2008). Leveling the playing field: Sincere and sophisticated players in the Boston mechanism. American Economic Review, 98(4), 1636-52.

Press and Information Office of the City of Münster. (2019). 3697 Kinder im Kita-Navigator vorgemerkt. Pressemitteilung der Stadt Münster. Retrieved 202110-10, from https://www.muenster.de/stadt/presseservice/ pressemeldungen/web/frontend/index.php? show=1010440

Roth, A. E. (1982). The economics of matching: Stability and incentives. Mathematics of Operations Research, 7(4), 617-628.

Roth, A. E., \& Peranson, E. (1999). The redesign of the matching market for American physicians: Some engineering aspects of economic design. American Economic Review, 89(4), 748-780.

Roth, A. E., \& Sotomayor, M. (1992). Two-sided matching. Handbook of Game Theory with Economic Applications, 1, 485-541.

Sönmez, T., \& Yenmez, M. B. (2020). Affirmative action with overlapping reserves. Working paper.

Veski, A., Biró, P., Pöder, K., \& Lauri, T. (2017). Efficiency and fair access in kindergarten allocation policy design. Journal of Mechanism and Institution Design, 2(1), 57-104.

Völker, K. (2018). Verdruss bei der Kitaplatz-Vergabe: Eltern drohen mit weiteren Klagen. Westfälische Nachrichten. Retrieved 2021-10-10, from https://www.wn.de/Muenster/3203115-Verdruss-bei-der -Kitaplatz-Vergabe-Eltern-drohen-mit-weiteren-Klagen 


\section{Appendix}

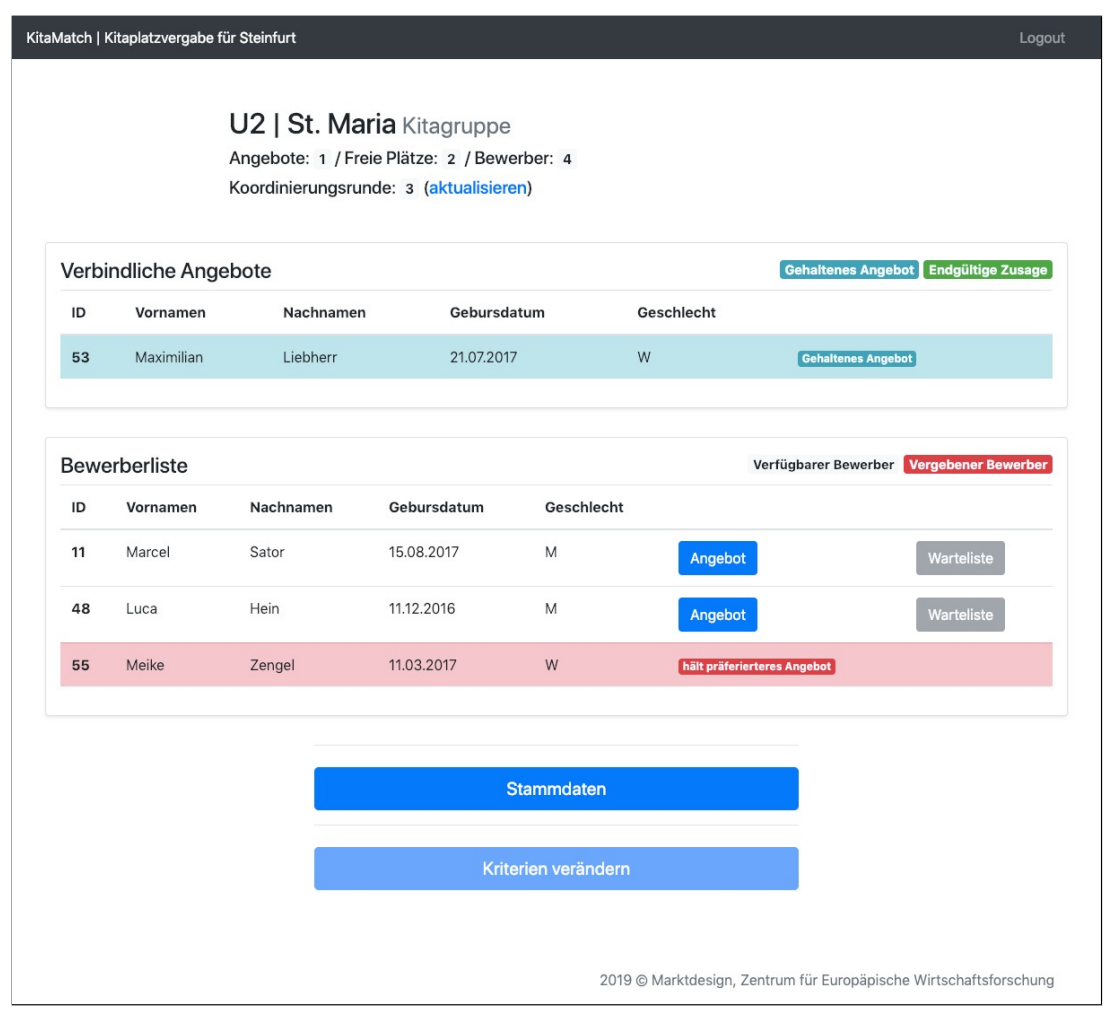

Figure 3: A screenshot of the Kitamatch application. In this view, childcare providers can register offers, they see the matches and rejections per round, and can modify their criteria catalog. The data presented here are anonymized. 


\begin{tabular}{ccccccc}
\hline Round & Age cohort & Program & Skipped child ID & Preferred child ID & Reason & \# Skipped \\
\hline 2 & U2 & 46 & 803 & 677 & gender & 3 \\
2 & U2 & 25 & 515 & 603 & - & 1 \\
2 & U2 & 28 & 522 & 500 & - & 3 \\
2 & U2 & 43 & 803 & 537 & - & 4 \\
2 & U2 & 46 & 803 & 746 & - & 8 \\
2 & U2 & 49 & 500 & 511 & - & 2 \\
2 & 2 & 65 & 210 & 297 & gender & 3 \\
2 & O3 & 33 & 33 & 56 & gender & 5 \\
2 & O3 & 33 & 33 & 37 & gender & 2 \\
2 & O3 & 45 & 12 & 33 & gender & 1 \\
2 & O3 & 12 & 88 & 103 & - & 1 \\
2 & O3 & 12 & 88 & 826 & - & 2 \\
2 & O3 & 75 & 831 & 48 & - & 1 \\
2 & O3 & 75 & 831 & 145 & - & 12 \\
3 & 2 & 41 & 359 & 454 & gender & 2 \\
3 & 2 & 65 & 359 & 381 & - & 2 \\
3 & O3 & 24 & 815 & 54 & age & 2 \\
4 & O3 & 24 & 815 & 836 & age & 1 \\
4 & O3 & 24 & 815 & 132 & age & 2 \\
\hline
\end{tabular}

Table 3: List of deviations of programs' sequence of offers (revealed preferences) from the pre-sorted ranking implied by the criteria catalog for the city of Greven. For each deviation, the table reports the ID of the first skipped child on the presorted ranking and the child ID that was preferred by the program. It also gives the reason and the total number of children skipped. Of the 19 deviations, 6 are to achieve gender balance and 3 to achieve heterogeneity in age. 


\begin{tabular}{cccc}
\hline Age cohort & Program & Excess capacity & Adjusted capacity \\
\hline U2 & 52 & 1 & 2 \\
2 & 29 & 1 & 6 \\
2 & 35 & 2 & 15 \\
2 & 41 & 1 & 7 \\
2 & 56 & 1 & 16 \\
2 & 62 & 1 & 6 \\
O3 & 24 & 1 & 13 \\
O3 & 42 & 1 & 1 \\
O3 & 75 & 6 & 15 \\
\hline
\end{tabular}

Table 4: Capacity adjustment for Greven. For administrative purposes, in the Greven match, nine programs deliberately stated a capacity in excess of their actual vacancies. The capacities entered by programs were thus adjusted by subtracting these excess capacities. 